\title{
New elmisaurine specimens from North America and their relationship to the Mongolian Elmisaurus rarus
}

Gregory F. Funston, Philip J. Currie, and Michael E. Burns

Acta Palaeontologica Polonica 61 (1), 2016: 159-173 doi:http://dx.doi.org/10.4202/app.00129.2014

New specimens from Canada confirm the presence of elmisaurines in North America and shed light on the relationship of Leptorhynchos elegans to Mongolian forms. These specimens have hindlimb elements previously unknown from elmisaurines in the Dinosaur Park Formation, including tibiae and pedal phalanges. Metatarsal anatomy is sufficiently different to merit a generic distinction from Elmisaurus rarus, and both can be distinguished from Caenagnathus collinsi Sternberg, 1940 and Chirostenotes pergracilis. Differences between these taxa include body size, degree of coossification of the tarsometatarsus, and development of cruciate ridges of the third metatarsal. Histological analysis confirms that these differences are not correlated with ontogenetic age of the specimens. The results support the informal separation of caenagnathids based on metatarsal structure, and allow comments on paleobiological differences between caenagnathids and oviraptorids.

Key words: Dinosauria, Oviraptorosauria, Caenagnathidae, Elmisaurinae, Campanian, Maastrichtian, Canada, Saskatchewan, USA, Montana.

Gregory F. Funston [funston@ualberta.ca], Philip J. Currie [pjcurrie@ualberta.ca], and Michael E. Burns [mburns@ualberta.ca], Biological Sciences CW405, University of Alberta, Edmonton, Alberta T6G 2E9, Canada.

This is an open-access article distributed under the terms of the Creative Commons Attribution License (for details please see creativecommons.org), which permits unrestricted use, distribution, and reproduction in any medium, provided the original author and source are credited. 
